\title{
3D Microstructural and Damage Analysis of Polymer Matrix Composites Using X-ray Computed Tomography with high contrast and submicron voxel resolution
}

\author{
J. Raghavan ${ }^{1}, \mathrm{SH} \mathrm{Lau}{ }^{2 *}$, Amir Asadi $^{1}$, Abhishek Gupta $^{1}$, Luke Hunter $^{2}$, Mike Boswick ${ }^{1}$ \\ ${ }^{1}$ Composite Materials and Structures Research Group \\ \& Department of Mechanical and Manufacturing Engineering, \\ University of Manitoba, Winnipeg, MB R3T 5V6, Canada \\ ${ }^{2}$ Xradia Inc, 5052 Commercial Circle, Concord, CA 94520, USA
}

Conventional optical and electron microscopy requires elaborate sample preparation and physical sectioning / chemical etching to expose sub-surface features. Furthermore, accurate modeling of 3D information from 2D images is difficult. X-ray computed tomography is a rapidly emerging 3-D imaging technique for non-destructive evaluation of biological and non-biological materials [1-5]. Microstructural features of interest at various size scales, in a polymer composite, are fiber and void volume fraction, fiber orientation and architecture, fiber-matrix interface, and various damage modes. These low $\mathrm{Z}$ materials coupled with small difference in density between the constituents of the polymer present significant challenges in imaging the various microstructural features within a thin polymer composite lamina layer of a composite laminate, because conventional X-ray detectors of microCT equipment lacks the contrast and resolution. While the contrast may be acceptable for low resolution features $(>5 \mathrm{um})$ focused in many publications on textiles and textile composites, higher contrast and resolution is required to detect damages, cracks or voids within these materials which are in the order of a few microns to sub-micron lengthscale. While resolution may be improved with submicron x-ray spot sized sources in conventional microCTs ( or nanoCTs), most of the resolution improvements are confined to $2 \mathrm{D}$ imaging, since higher resolution 3D imaging in the submicron resolution regime places tremendous penalty on sample sizes, thickness and working distance. In this study, a microCT equipment capable of submicron pixel resolution and improved contrast resolution, is used to study the multi-scale microstructure and damage in non-woven and woven continuous fiber and random fiber composites. Resolution of cracks or features as small as 1 to 3 microns in a composite material in relatively large sample of several $\mathrm{cm}$ dimension is also possible, making this technique advantageous to study materials AS IS, without much sample trimming or destruction. Some representative images are presented in Figures 1-5. Issues related to non-destructive microstructural and damage analysis of the polymer composites will be highlighted and discussed during presentation.

References:

1. J. Adrien, E. Maire, N. Gimenez, V. Sauant-Moynot, Acta Materialia, 55(2007)1667-79

2. A. Alemdar, H. Zhang, M.Sain, G. Cescutti, J. Mussig, Advanced Engineering Materials, 10(2008)126130

3. S.V. Lomov, D.S. Ivanov, T.C. Truong, I. Verposet, F. Baudry, K. Vanden Bosche, H. Xie, Composite Science and Technology, 68 (2008) 2340-49

4. P. Badel, E. Vidal-Salle, E. Maire, P. Boisse, Composite Science and Technology, 68 (2008) 2433-40

5. P.J. Schilling, BPR Kharedla, AK Tatiparthi, M.A. Verges, PD Herrington, Composite Science and Technology, 65 (2005)2071-78 


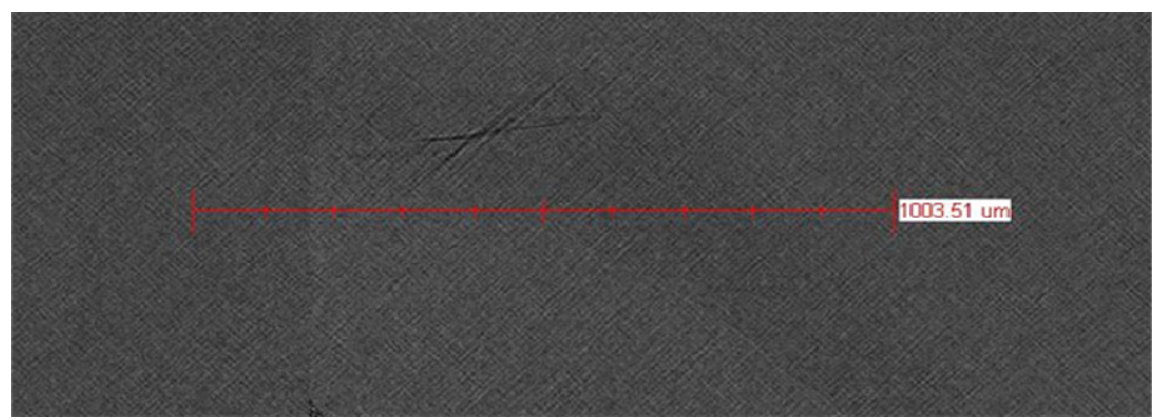

Figure 1. 2-D mosaic image showing damage in $[+45,-45]$ textile laminate

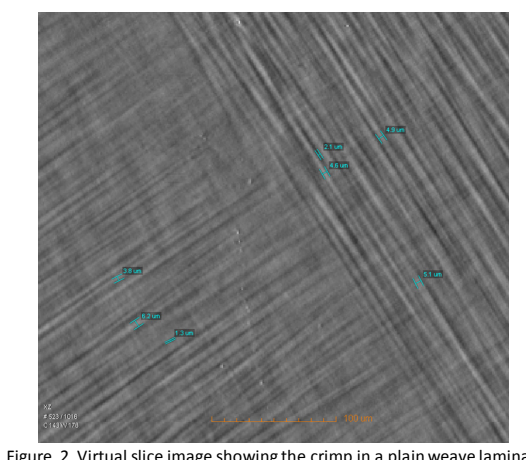

Figure 2. Virtual slice image showing the crimp in a plain weave laminate; individual Carbon fibers and spacing bewteen them are visible

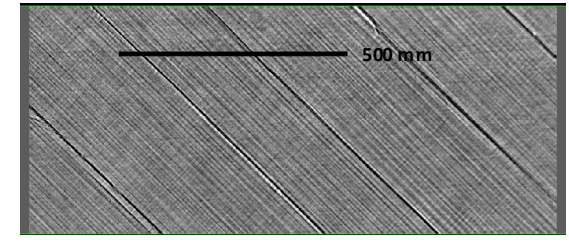

Figure 4. Virtual slice showing damage in [45] layer of a quasi-isotropic laminate

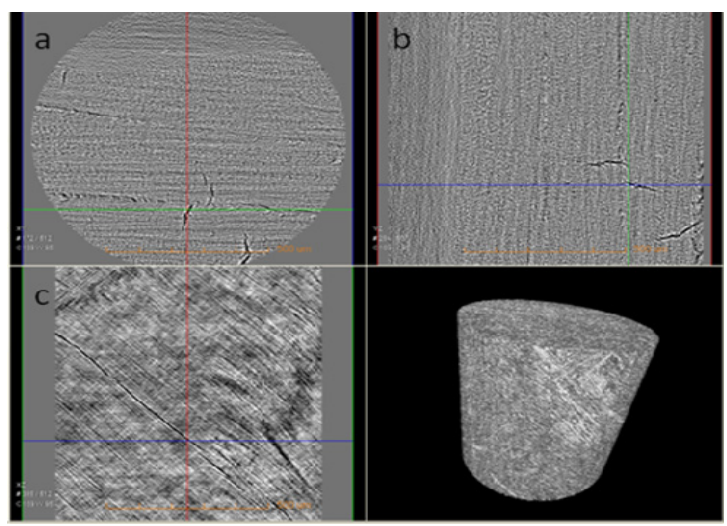

Figure 3. Damage within a yarn of $[+45,-45]$ textile la minate; $a, b, c$ are top edgo, side edge and front cf the slice of the yarn; cracking at the crimp is observed in $c$
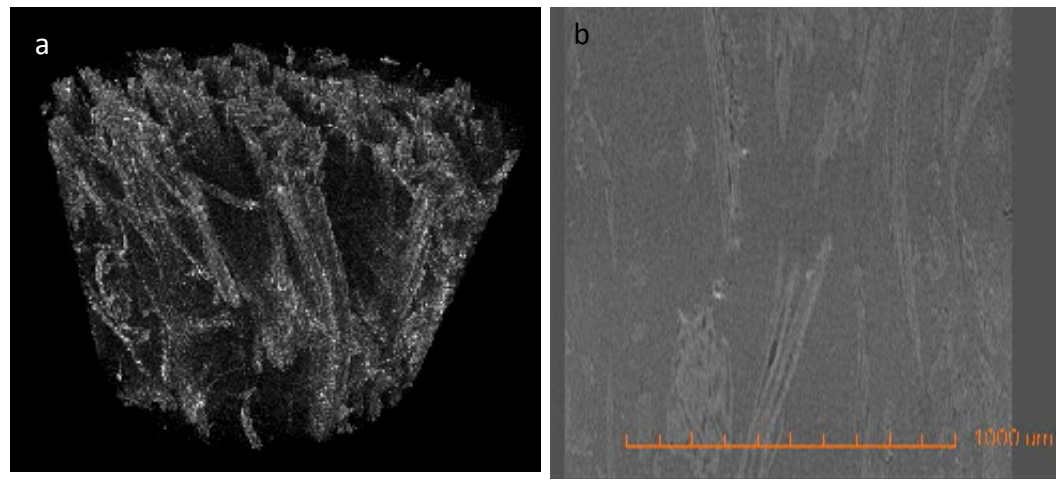

Figure 5. a-reconstructed 3-D image of a hemp fiber mat; b- biopolymer/hemp composite 